\title{
Functional collagen conduits combined with human mesenchymal stem cells promote regeneration after sciatic nerve transection in dogs
}

\author{
Yi Cui ${ }^{1,2^{*}}$ | Yao Yao ${ }^{3^{*}} \mid$ Yannan Zhao ${ }^{2^{*}}$ | Zhifeng Xiao ${ }^{2}$ | Zongfu Cao ${ }^{1}$ | Sufang $\mathrm{Han}^{2}$ | \\ Xing $\mathrm{Li}^{2}$ | Yong Huan ${ }^{4}$ | Juli Pan $^{3}$ | Jianwu Dai ${ }^{2}$ (D)
}

\author{
${ }^{1}$ Reproductive and Genetic Center of National \\ Research Institute for Family Planning, Beijing, \\ China \\ ${ }^{2}$ Key Laboratory of Molecular Developmental \\ Biology, Institute of Genetics and \\ Developmental Biology, Chinese Academy of \\ Sciences, Beijing, China \\ ${ }^{3}$ Department of Prosthodontics, School of \\ Stomatology, Capital Medical University, \\ Beijing, China \\ ${ }^{4}$ State Key Laboratory of Nonlinear Mechanics \\ (LNM), Institute of Mechanics, Chinese \\ Academy of Sciences, Beijing, China \\ Correspondence \\ Jianwu Dai, Institute of Genetics and \\ Developmental Biology, Chinese Academy of \\ Sciences, 3 Nanyitiao, Zhongguancun, Beijing \\ 100190, China. \\ Email: jwdai@genentics.ac.cn \\ Juli Pan, Department of VIP Service, School of \\ Stomatology, Capital Medical University, \\ Beijing 100190, China. \\ Email: panjuli@163.com \\ Funding information \\ Youth Innovation Promotion Association, CAS, \\ Grant/Award Number: 2016096; National Key \\ Research and Development Project, Grant/ \\ Award Numbers: 2016YFC1101500 and \\ 2016YFC1000800; Key Research Program of \\ the Chinese Academy of Sciences, Grant/ \\ Award Number: ZDRW-ZS-2016-2
}

\begin{abstract}
Numerous studies have focused on the development of novel and innovative approaches for the treatment of peripheral nerve injury using artificial nerve guide conduits. In this study, we attempted to bridge $3.5-\mathrm{cm}$ defects of the sciatic nerve with a longitudinally oriented collagen conduit (LOCC) loaded with human umbilical cord mesenchymal stem cells (hUC-MSCs). The LOCC contains a bundle of longitudinally aligned collagenous fibres enclosed in a hollow collagen tube. Our previous studies showed that an LOCC combined with neurotrophic factors enhances peripheral nerve regeneration. However, it remained unknown whether an LOCC seeded with hUC-MSCs could also promote regeneration. In this study, using various histological and electrophysiological analyses, we found that an LOCC provides mechanical support to newly growing nerves and functions as a structural scaffold for cells, thereby stimulating sciatic nerve regeneration. The LOCC and hUC-MSCs synergistically promoted regeneration and improved the functional recovery in a dog model of sciatic nerve injury. Therefore, the combined use of an LOCC and hUC-MSCs might have therapeutic potential for the treatment of peripheral nerve injury.
\end{abstract}

\section{KEYWORDS}

dog, human umbilical cord mesenchymal stem cells (hUC-MSCs), longitudinally oriented collagen conduit (LOCC), nerve regeneration, sciatic nerve

\section{1 | INTRODUCTION}

Peripheral nerve injuries are often caused by trauma or surgery for diseases of the peripheral nervous system. The peripheral nervous system has the capacity for regeneration after injury. However, the regenerating axons are often misdirected and do not reach their target, resulting in poor functional recovery. In the operative treatment of peripheral nerve injury, the nerve stumps can be anastomosed directly for short-distance nerve injuries (i.e., shorter than $5 \mathrm{~mm}$ ). When the size of the defect exceeds $5 \mathrm{~mm}$, it is necessary to bridge the nerve

*These authors contributed equally to this work. stumps by autograft or the use of graft materials. As the preferred treatment strategy, autologous nerve grafting is commonly used clinically to repair peripheral nerve defects (Matsumoto et al., 2000). However, autografts have several drawbacks, such as the lack of graft material, mismatch in nerve size, and the formation of painful neuromas (Lundborg, Dahlin, Dohi, Kanje, \& Terada, 1997). As a consequence, a great deal of effort has been put into the development of artificial nerve conduits to replace autologous nerve grafts for the treatment of peripheral nerve injury.

Tissue-engineered nerve guide conduits have many advantages over autografts, including no donor site morbidity, ease in size matching, and no length limitation (Ao et al., 2011). An increasing 
number of researchers are working on developing novel biological materials, especially in combination with support cells or growth factors, to stimulate regeneration over longer distances (Carriel et al., 2013; Cui, Lu, et al., 2014; Gu, Ding, \& Williams, 2014). Our previous studies showed that longitudinally oriented collagen conduit (LOCC) can resist deformation during implantation and degrade progressively during the following 6 months of regeneration (Ma et al., 2014). The LOCC manufactured by our lab displays biocompatibility, biodegradability, and low toxicity to nerve fibres. Previous studies showed that an LOCC combined with neurotrophic factors promoted regeneration in both the spinal cord and peripheral nervous system in animal models (Faroni, Mobasseri, Kingham, \& Reid, 2015; Han et al., 2015; Lu et al., 2015). Here, we developed a novel procedure to evaluate whether the combined use of an LOCC and human umbilical cord mesenchymal stem cells (hUC-MSCs) facilitates peripheral nerve regeneration to a greater extent than an LOCC alone.

To promote neuronal outgrowth and accelerate axonal regeneration, many types of seed cells, such as Schwann cells, mesenchymal stem cells (MSCs), and embryonic stem cells, have been used in combination with tissue-engineered nerve guide conduits. Of these cell types, MSCs have the fewest problematic ethical concerns and the lowest risk of teratoma formation compared with other stem cells, such as embryonic stem cells and induced pluripotent stem cells (Kao et al., 2015). In comparison with bone marrow stem cells and adipose-derived mesenchymal stem cells, hUC-MSCs possess greater paracrine effects and have higher proliferative and differentiation potentials (Horwitz \& Dominici, 2008). Accumulating evidence suggests that hUC-MSCs promote axon regeneration in the peripheral nervous system following peripheral nerve injury (Carriel, Alaminos, Garzon, et al., 2014; Wakao, Matsuse, \& Dezawa, 2014; Zarbakhsh, Goudarzi, Shirmohammadi, \& Safari, 2016). For example, transplantation of hUC-MSCs promotes axonal regeneration and functional recovery after sciatic nerve transection injury (Guo et al., 2015). Additionally, hUC-MSCs possess immunosuppressive properties that curtail allogeneic or xenogeneic immune responses in animal models after grafting (Lee et al., 2014; Tsai et al., 2009; Wang et al., 2014).

In this study, we evaluated the cellular biocompatibility of the LOCC. We then investigated whether hUC-MSCs seeded onto the LOCC promote neural regeneration after long-segment peripheral nerve injury in a large animal model, the dog. To better evaluate the long-term impact of the LOCC on sciatic nerve regeneration, dogs were sacrificed for analyses 9 months after surgery, longer than in previous studies. Our results suggest that the LOCC helps to localize hUC-MSCs to the injury site and also guides the regenerating nerve to grow in an orderly manner across the defect. In addition to serving a structural role, the guidance channel created by the LOCC may also prevent the formation of fibrous scar tissue that would otherwise hinder nerve regeneration. The orderly arrangement of longitudinally aligned collagenous fibres (LOCs) may guide the nascent nerve fibres to grow from the proximal end of the injured site to the end of the distal stump. Our long-term study demonstrated that the combination of hUC-MSCs and an LOCC promoted sciatic nerve regeneration and functional recovery.

\section{2 | MATERIALS AND METHODS}

\section{1 | Preparation of tissue-engineered collagen nerve conduits}

The LOCC was prepared as described previously (Cao et al., 2013). The hollow collagen nerve conduit was created by wrapping the collagen membrane (provided by Yantai Zhenghai Bio-tech Co., Ltd) around a cylindrical tube made of glass. The hollow collagen conduit was cross-linked with cross-linking solution-30 mM 1-(3dimethylaminopropyl)-3-ethylcarbodiimide hydrochloride and $10 \mathrm{mM}$ $\mathrm{N}$-hydroxysuccinimide in $50 \mathrm{mM}$ 2-(N-morpholino)-ethanesulfonic acid solution, $\mathrm{pH}$ 5.5-and then lyophilized. The LOCC had a diameter of $5 \mathrm{~mm}$ and a wall thickness of approximately $60 \mu \mathrm{m}$. The collagen tubes were cut into 4-cm-long pieces, which were used to bridge the nerve gap. Before grafting, a bundle of LOCs containing 30 collagen fibres was inserted into the hollow collagen nerve conduit. The dimension of one LOC fibre was 200-400 $\mu \mathrm{m}$. LOCs were prepared from bovine aponeurosis as previously described (Cao et al., 2013). In brief, tissues from fresh white bovine aponeurosis, including the residual muscles, connective tissues, and fat were removed. Thereafter, the cellular components were removed by treatment with $1 \%$ tri(n-butyl) phosphate (Aldrich, Germany) for $48 \mathrm{hr}$ at $4{ }^{\circ} \mathrm{C}$ and subsequently rinsed repeatedly in distilled water to completely remove residual matter. The LOCs were then separated from the processed aponeurosis and freeze-dried.

The mechanical properties of the transversal form of LOCC were measured using an Instron E1000 apparatus (Instron, Canton, MA). The compression test was performed with increasing forces, and the displacements were recorded continuously every $0.1 \mathrm{~s}$ (Dwyer et al., 2016; Grasman, Pumphrey, Dunphy, Perez-Rogers, \& Pins, 2014). During each test, the machine recorded elapsed time, tensile force, and tensile displacement at 600 points per second. The force-displacement curves were generated from the test data. The surface structure of the LOCs was observed under a scanning electron microscope (S-3000N, Hitachi, Tokyo, Japan).

\subsection{Animals and surgical procedures}

Twenty adult Beagles (15 months, $9 \mathrm{~kg}$ ) were purchased from the Beijing Laboratory Animal Research Centre (Beijing, China). The dogs were kept in cages measuring $110 \times 75 \times 80 \mathrm{~cm}$. All animal procedures were performed in accordance with Chinese Ministry of Public Health Guide and U.S. National Institute of Health Guide for the care and use of laboratory animals and was approved by the Institutional Animal Care and Use Committee Bioethics Committee of the Institute of Genetics and Developmental Biology, Chinese Academy of Sciences. Animals (of either sex, $n=5$ in each group) were randomly divided into the following four groups: negative control, LOCC, hUC-MSC in combination with LOCC (LOCC/hUC-MSC), and autograft (positive control) groups. Animals were kept separately in cages at a controlled temperature $\left(20-24{ }^{\circ} \mathrm{C}\right)$ under a $12-\mathrm{hr}$ light/dark cycle. Dogs were allowed free access to water and food.

Animals were anaesthetized by intramuscular administration of $10 \mathrm{mg} / \mathrm{kg}$ ketamine and $2 \mathrm{mg} / \mathrm{kg}$ xylazine. The left gluteal muscle 
was dissected to expose the sciatic nerve, and then, a 35-mm-long segment of sciatic nerve was removed using microsurgical scissors. The proximal and distal nerve endings were connected via a 4-cm-long nerve conduit in the LOCC and LOCC/hUC-MSC groups. Before grafting, the LOCC was flushed with phosphate buffered saline solution. In the LOCC/hUC-MSC group, $1 \times 10^{6}$ hUC-MSCs were seeded onto the LOCs an hour before grafting to the site of injury. In the autograft group, the orientation of the excised sciatic nerve segment was reversed before reimplantation. In the negative control group, only the muscle and skin were sutured, whereas the nerve gap remained untouched. After surgery, the animals received conventional anti-inflammatory treatment for 2 weeks, whereas they did not receive immunosupressive treatments.

\section{3 | Cell culture}

Isolation and verification of hUC-MSCs were carried out as previously described (Sun et al., 2016; Zarbakhsh et al., 2016). The identification of hUC-MSCs has been standard in our laboratory and has proven reliable (Sun et al., 2016). Cells possessed a proliferative ability of 10 passages in culture medium. Cells from passages 3 or 4 were used in the in vivo study. Cells were cultured in Mesenchymal Stem Cell Medium (ScienCell, Carlsbad, CA). When cells reached $80 \%$ confluence, they were treated with $0.25 \%$ trypsin before the graft procedure.

\section{4 | Electrophysiological measurements}

The sciatic nerve at the site of injury was re-exposed under anaesthesia 9 months after surgery. An electrode was inserted into the main sciatic nerve trunk proximal, medial, or distal to the site of injury (electrical stimuli were given at $5 \mathrm{~mA}$ ). Compound muscle action potentials (cMAPs) were recorded from the ipsilateral gastrocnemius belly. Concurrently, normal cMAPs were measured from the contralateral, uninjured side. The stimulations were performed in triplicate, with a time interval of $1 \mathrm{~s}$ to allow the nerve and muscle to relax. Histograms were plotted of the ratio of cMAPs on the ipsilateral injured side to those on the contralateral normal side $(\mathrm{CMAP}$ ratio $=\mathrm{CMAP}$ of injured side/cMAP of healthy side $\times 100 \%$ ). Quantification of cMAP amplitude, latency, and area under the rectified curve were performed using an electrophysiology system (RM6240, Chengdu, China).

\section{5 | Electron microscopy}

Tissue samples were first fixed in $2.5 \%$ glutaraldehyde and then fixed in $1 \%$ osmic acid solution. Samples were dehydrated through a graded ethanol series before embedding. Next, samples were cut into 50-nmthick sections and stained with lead citrate and uranyl acetate. Finally, samples were imaged on a transmission electron microscope (H-7650B, HITACHI, Japan). The G-ratio of axons was calculated by the inner diameter of the axon divided by the fibre diameter.

HUC-MSCs seeded onto LOCs were fixed in $2.5 \%$ glutaraldehyde and then dehydrated with ethanol and isoamyl acetate. Samples were dried in a critical point drier (Hitachi, Tokyo, Japan) for gold coating. Images were captured using a scanning electron microscope (S-3000N; Hitachi, Tokyo, Japan).

\section{6 | Histological analyses}

Regenerated tissues were fixed in $4 \%$ paraformaldehyde and then embedded in paraffin. Haematoxylin-eosin (HE) staining, immunohistochemistry, and MCOLL histochemical staining were performed to observe the histomorphology of the sciatic nerve. All images were captured on an inverted phase contrast microscope $(\times 200)$. The specimens were cut into sections of 4-5 $\mu \mathrm{m}$ thickness on polylysine-coated slides, followed by paraffin imbedding. For HE staining, the slides were dewaxed with xylene, dehydrated with a graded ethanol series, and stained with haematoxylin for $5 \mathrm{~min}$. Next, the slides were immersed in $1 \%$ hydrochloric acid for $2 \mathrm{~s}$ and then stained with eosin for $5 \mathrm{~min}$. Finally, the sections were dehydrated and mounted with neutral resin. Immunohistochemical analysis was performed using a kit (HistostainPlus IHC Kit, Neobioscience, China) according to the manufacturer's instruction. Tissue slides were dewaxed with xylene and rehydrated through a graded alcohol series. After antigen retrieval, slides were incubated with $3 \%$ hydrogen peroxide for $10 \mathrm{~min}$. The primary antibodies were as follows: anti-GAP-43 (1:1,000; NB300-143, Novus, Sunnyvale, CA, USA), anti-Neurofilament (NF; 1:1,000; ab7795, Abcam) and antiS100 (1:1,000; ab41548, Abcam; Carriel et al., 2013; Carriel, Garzon, Alaminos, et al., 2014; Carriel, Garzon, Campos, Comelissen, \& Alaminos, 2014).

The MCOLL histochemical staining assay can be used to quantify the amount of regenerated myelin (Carriel, Campos, Alaminos, Raimondo, \& Geuna, 2017; Carriel, Garzon, Alaminos, \& Campos, 2011). Briefly, tissue slides were dewaxed, rehydrated, and incubated in Luxol fast blue solution (Solvent Blue 38; S3382, Sigma) at $56^{\circ} \mathrm{C}$ for $16 \mathrm{hr}$. Next, the slides were incubated in $0.05 \%$ lithium carbonate for $30 \mathrm{~s}$ and rinsed in $70 \%$ alcohol until myelinated and unmyelinated regions were distinguishable. Then, sections were incubated in $0.2 \%$ sirius red F3B stain (C.I. 35780, Sigma-Aldrich) for $30 \mathrm{~min}$ at room temperature.

Masson trichrome (MT) staining was performed using a kit (cat\# G1340, Solarbio Life Sciences, Beijing, China) according to the manufacturer's protocol. Gastrocnemius muscle tissues were fixed in $4 \%$ paraformaldehyde and then embedded in paraffin. The paraffinembedded muscle tissues were sectioned into 5- $\mu \mathrm{m}$-thick sections. Images of the stained sections were captured using a microscope at a magnification of $\times 200$.

To perform relative quantitative analysis of the immunohistochemistry image, the representative images of each animal in each group $(n=3$ ) were analysed using Image-Pro Plus software 6.0 (Media Cybernetics, Silver Spring, MD, USA; Carriel et al., 2013; Carriel, Garzon, Alaminos et al., 2014). In brief, the Image-Pro Plus software was started and then click "Count/Size" in Measure menu and choose "Manual" to select colours. Then, the positive area was selected, and the results can be found in "Measurement Data." The percentage of GAP-43-positive, NF-positive, and S100-positive areas were calculated as the ratio of the positively stained area to the total area. The same method was used to quantify the positive (blue) area in MCOLL-stained sections. The intensity of immunohistochemical staining was calculated as the ratio of the integrated optical density to the area. The average muscle fibre cross-sectional area $\left(\right.$ CSA; $\left.\mu m^{2}\right)$ of the gastrocnemius muscle was calculated following MT staining as follows: CSA $\%=$ positively stained muscle fibre area/total area $\times 100 \%$. 
(a)

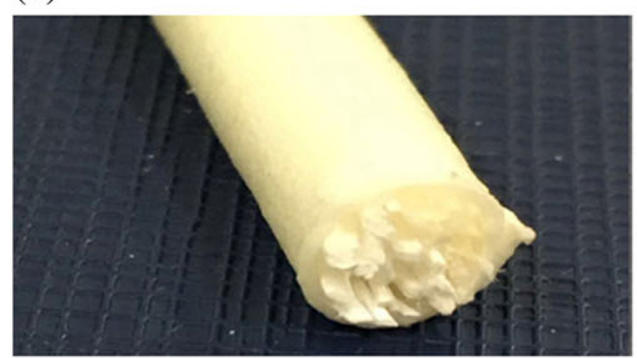

(c)

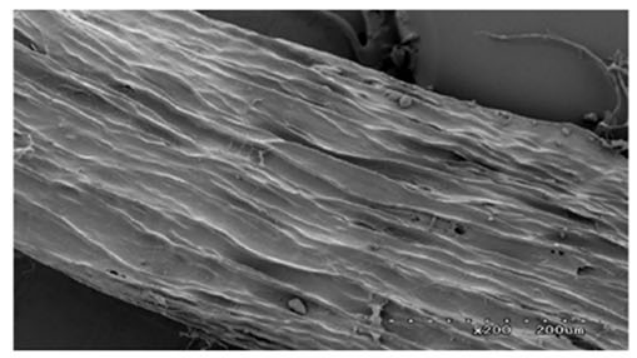

(b)

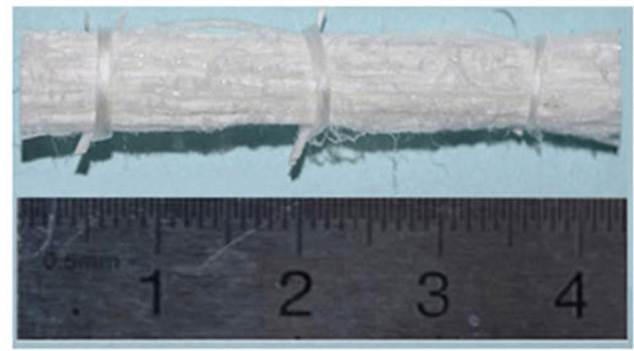

(d)

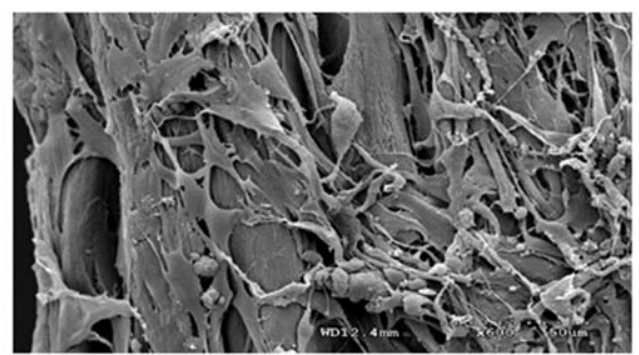

(e)
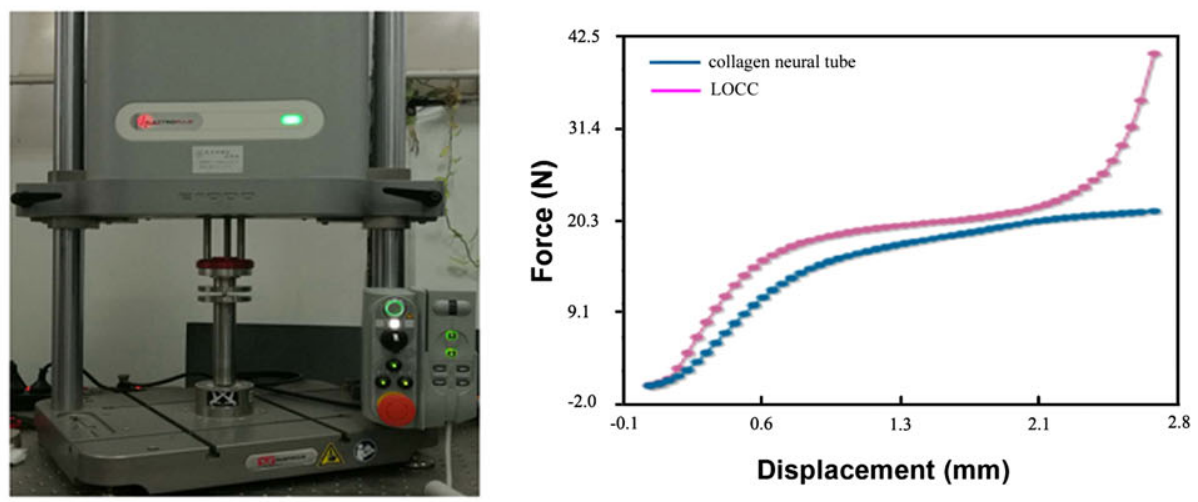

(f)

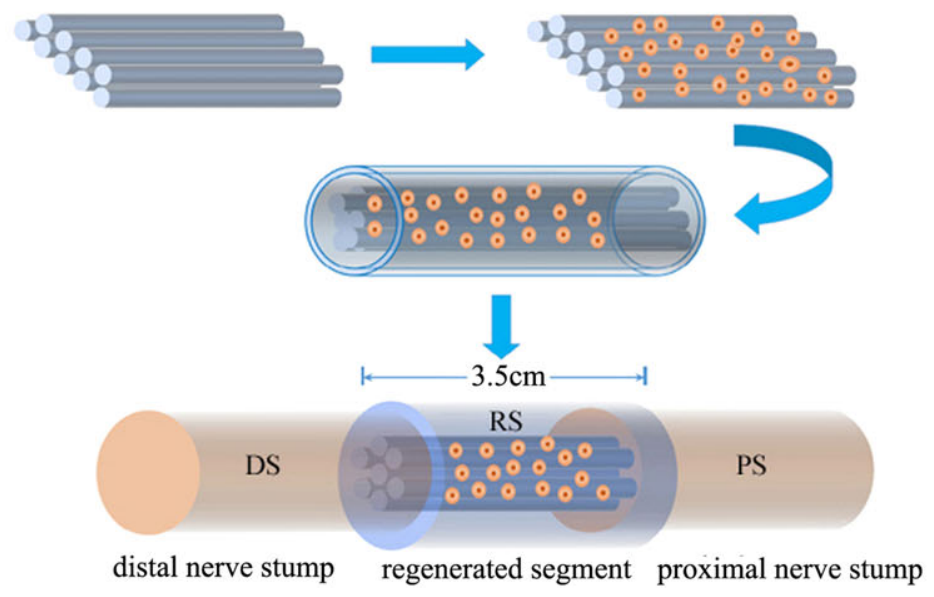

FIGURE 1 General and morphological observations of the longitudinally oriented collagen conduits (LOCCs). The schematic diagram shows the process underlying the repair of the experimental sciatic nerve injury with the combination of the LOCCs and human umbilical cord mesenchymal stem cells (hUC-MSCs): (a) general observations of the LOCCs; (b) general observations of the longitudinally aligned collagenous fibres (LOCs); (c) representative scanning electron microscopic images of the LOCs; (d) representative scanning electron microscopic images of the hUC-MSCs cultured on the LOCs; (e) the ability of the LOCCs to resist mechanical stress; and (f) a flow chart of the LOCC/hUC-MSC treatment procedure [Colour figure can be viewed at wileyonlinelibrary.com] 


\section{7 | Muscle mass analysis}

Peripheral nerve functional recovery is also reflected as a change in the weight ratio of the gastrocnemius muscles. The mid-belly regions of the ipsilateral (injured) and the contralateral (opposite) gastrocnemius muscles were dissected and weighed while still wet using an electronic balance. The relative muscle weight was calculated as the weight of the injured side divided by the weight of the uninjured side.

\section{8 | Data analysis}

All data were reported as means and standard deviations. The ShapiroWilk test was performed to check the normality of all the variables. Pairwise differences and $95 \%$ confidence intervals were assessed by one-way analysis of variance with Tukey's Honest Significant Difference test. All data analyses were performed using R3.3.2 software.

\section{3 | RESULTS}

\section{1 | Gross morphology of LOCC and hUC-MSCs cultured on LOCC}

As shown in Figure 1, the natural collagen LOCC (Figure 1a) consists of a bundle of LOCs (Figure $1 \mathrm{~b}$ ) and a collagen nerve conduit. The average diameter of one collagen scaffold fibre was about $500 \mu \mathrm{m}$. Scanning electron microscopic analysis showed that the surface of the LOCs displayed a longitudinally aligned arrangement. Thus, the LOC morphology encouraged regenerating nerve fibres to grow in an orderly manner from the proximal to the distal nerve ends (Figure 1c). Furthermore, the seeded hUC-MSCs adhered well to the LOCs and exhibited a normal cellular morphology. LOCs showed good biocompatibility with hUC-MSCs. Furthermore, as shown in Figure 1d, the hUC-MSCs were attached to the surface of the LOCs tightly. These findings suggest that LOCs are capable of localizing the seeded hUC-MSCs to the damaged nerve, preventing them from spreading to the surrounding tissue. The mechanical test showed that LOCCs possess a degree of rigidity, which likely helps to resist collapse during neural regeneration (Figure 1e). Given these advantageous characteristics of the LOCs, we loaded hUC-MSCs onto LOCs to create an LOCC/hUC-MSCs graft for the treatment of long (3.5 cm long) sciatic nerve defects in the dog (Figure 1f).

\subsection{General observations of the regenerating sciatic nerve}

Immediately after sacrificing the animals, the grafted LOCC together with the proximal and distal ends of the sciatic nerve was harvested for observation. As shown in Figure 2, the LOCC possessed sufficient mechanical strength to resist the mechanical stress from neighbouring tissues. After the LOCC was grafted into the injury site, white linear tissue replaced the collagen tube. The newly grown, nerve-like tissue, which bridged the sciatic nerve gap, was well attached to the LOCC. In parallel, the LOCC gradually degraded. No obvious inflammatory reaction or tissue swelling was observed around the grafted LOCC neural tube in the LOCC grafted animals. The results demonstrate that the relatively sealed LOCC helped prevent fibrous scar tissue from accumulating at the site of injury and avoided the formation of neurofibroma. Therefore, the LOCC might function as a guide and bridge for regenerating fibres, helping them reach the distal nerve end.

(a)
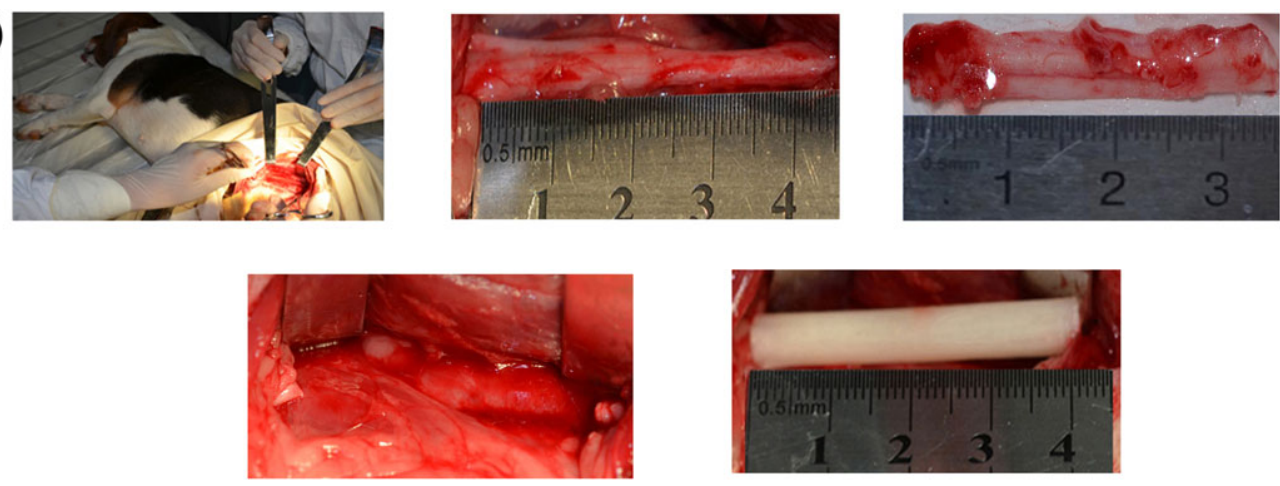

(b)

LOCC

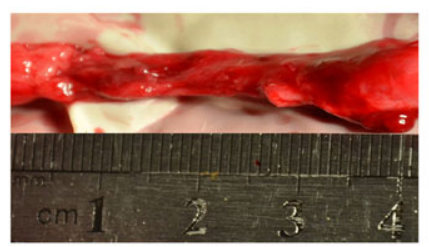

LOCC/hUC-MSCs

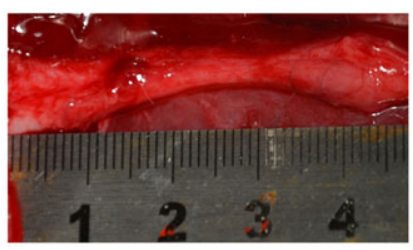

autograft

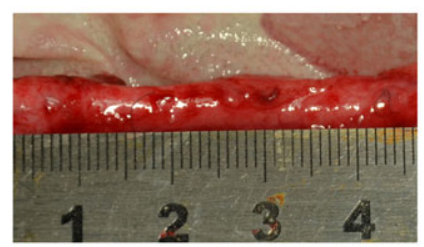

FIGURE 2 (a) Surgical procedures. The sciatic nerve of the dog was completely exposed after cutting through the skin and muscle. The sciatic nerve segment was then removed, leaving a 35-mm-long gap after retraction of the nerve stump. In the longitudinally oriented collagen conduit (LOCC) and LOCC/human umbilical cord mesenchymal stem cell (hUC-MSC) groups, the LOCC was sutured between the two nerve stumps. (b) General observations of the regenerated nerve-like tissue segment in each of the experimental groups 9 months after surgery [Colour figure can be viewed at wileyonlinelibrary.com] 
(a) $\mathrm{NC}$

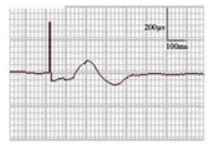

LOCC/hUC-MSCs
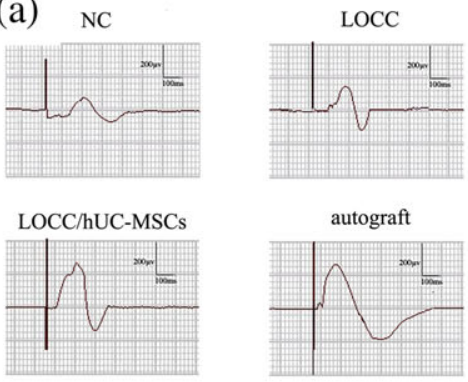

(b) $\mathrm{NC}$

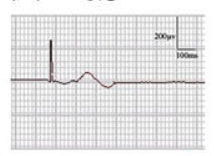

LOCC/hUC-MSCs

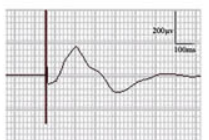

(c) $\mathrm{NC}$
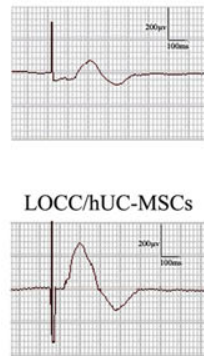

LOCC

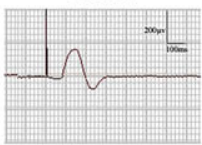

autograft

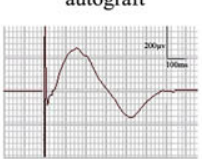

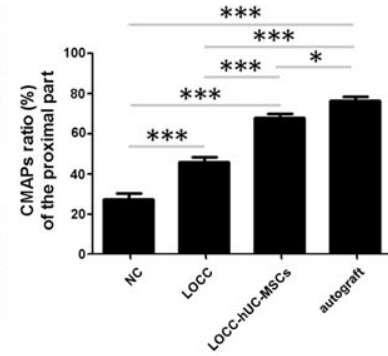
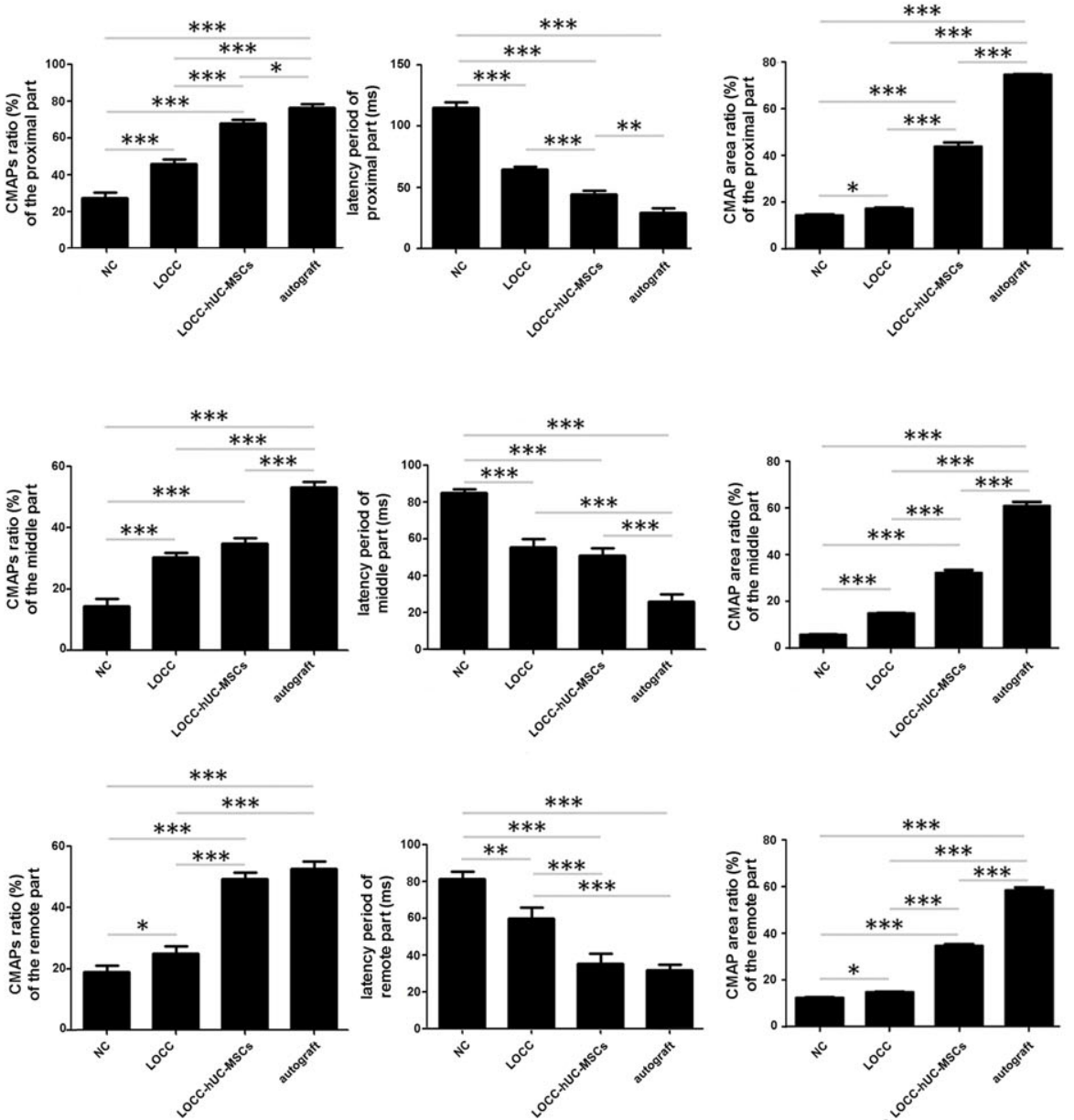

FIGURE 3 Quantitative results of the electrophysiological evaluation made 9 months after surgery. Representative measurements of the (a) proximal, (b) middle, and (c) distal sections of the injured canine sciatic nerve. The data are shown as the mean \pm standard deviation. ${ }^{*} p<.05$, ${ }^{* *} p<.01$, or ${ }^{* * *} p<.001$, compared with the negative control (NC) group. cMAPs = compound muscle action potentials; hUC-MSCs = human umbilical cord mesenchymal stem cells; LOCC = longitudinally oriented collagen conduit [Colour figure can be viewed at wileyonlinelibrary.com]

$\mathrm{NC}$

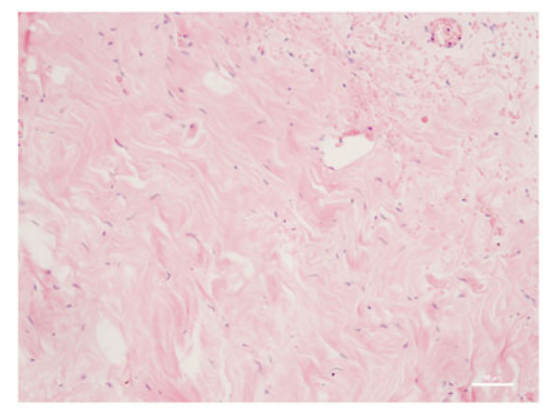

LOCC/hUC-MSCs

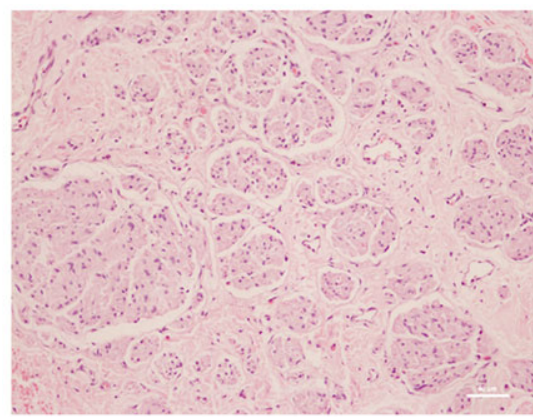

\section{LOCC}

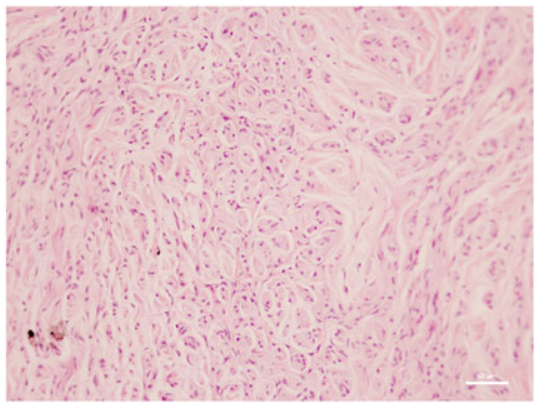

autograft

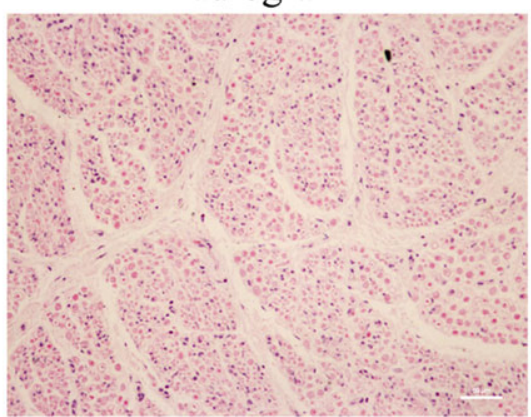

FIGURE 4 Representative transverse sections of the central part of the regenerated sciatic nerve 9 months after surgery in the negative control (NC), longitudinally oriented collagen conduit (LOCC), LOCC/human umbilical cord mesenchymal stem cell (hUC-MSC) and autograft groups (haematoxylin-eosin staining) [Colour figure can be viewed at wileyonlinelibrary.com] 


\section{3 | Electrophysiological assessment}

The ratio of cMAP amplitudes and the area under the rectified curve between the injured and healthy sides were calculated 9 months after surgery, before sacrificing the animals. The CMAP amplitude ratio was significantly higher in the LOCC/hUC-MSCs compared with the LOCCalone group at three separate locations on the injured sciatic nerve. Among the groups, the autograft group had the highest ratios, whereas the negative control group had the lowest ratios (Figure 3). The cAMP latencies were shorter in the LOCC/hUC-MSCs group compared with the LOCC group. The negative control group showed the longest latencies. Taken together, the electrophysiological results indicated that nerve conduction was better in the LOCC/hUC-MSCs group than in the LOCC-alone group. Thus, the combination of hUC-MSCs and the LOCC promotes effective recovery of nerve conduction function.

\subsection{In vivo evaluation of sciatic nerve regeneration}

Nine months after surgery, the connective tissue, which joined to the nerve stump at the injury site, was harvested for histochemical analysis. Representative HE-stained transverse sections of the sciatic nerve are shown in Figure 4. Robust expression of GAP-43, NF, and S-100 proteins was observed in cross sections of regenerated sciatic nerve segments. As shown in Figure 5, GAP-43-positive, NF-positive, and $\mathrm{S}-100$-positive cells were detected in the middle part of the regenerated segment in the LOCC and LOCC/hUC-MSCs group. Moreover, GAP-43-positive, NF-positive, and S-100-positive cells showed similar distribution patterns and percentages. Additionally, labelling for these proteins revealed newborn nerve fibres traversing the middle part of the regenerated nerve segment in the LOCC and LOCC/hUC-MSC groups. GAP-43, NF, and S-100 labelling was more widely distributed in the LOCC/hUC-MSCs groups compared with the LOCC group
(Figure 5). GAP-43, NF, and S-100 were not detected in the negative control group. The results of the relative intensity in all groups showed the same tendency as the results of the relative positive area.

Given that myelin sheath formation is an important indicator of nerve regeneration, the G-ratio of myelinated fibres indicates better recovery of nerve function (Isaacs, Mallu, \& Batchelor, 2014; Li et al., 2017). The existence of myelinated nerve fibres was confirmed by MCOLL histochemistry (Figure 6) and transmission electron microscopy (Figure 7). MCOLL histochemistry results revealed a more intense positive signal in the middle portion of the segment in the LOCC/hUCMSCs group compared with the LOCC-alone group. Thickness and size of the regenerated myelinated fibres in the middle part of the segment were quantified using transmission electron microscopy. The G-ratios were as follows: $83.95 \pm 3.63$ in the LOCC group; $68.67 \pm 1.96$ in the LOCC/hUC-MSC group; and $56.21 \pm 4.12$ in the autograft group. The G-ratio results show that the LOCC/hUC-MSC group had thicker myelin sheaths on the regenerated nerve fibres at the midpoint of the nerve segment compared with the LOCC group.

\subsection{Assessment of gastrocnemius muscle properties in different treatment groups}

Gastrocnemius muscle function indirectly reflects sciatic nerve function. Therefore, we performed MT staining on the muscle. Before staining, the mean ratio of the gastrocnemius muscle weight was calculated. There was a statistically significant difference between the muscle weight ratios among the various groups. Weight loss in the gastrocnemius muscle was alleviated by local administration of hUC-MSCs. The net weight of gastrocnemius muscle was lower in the LOCC group than in the LOCC/hUC-MSCs group (Figure 8a). MT staining showed that the area of the gastrocnemius muscle fibre (a)

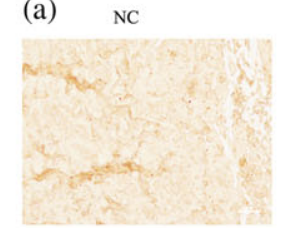
LOCC/hUC-MSCs
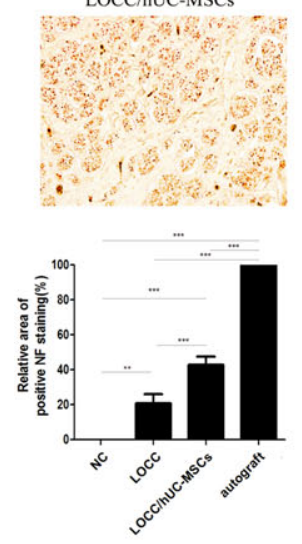

LOCC

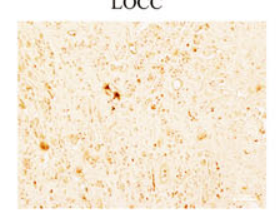

autograft
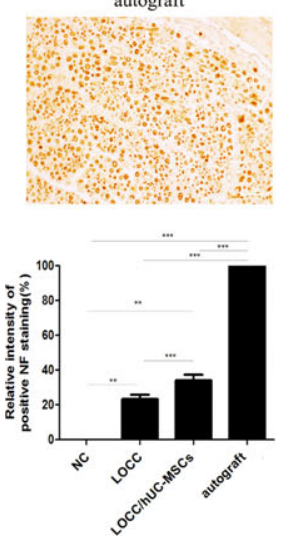

(b)

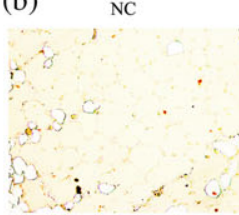

LOCC/hUC-MSCs
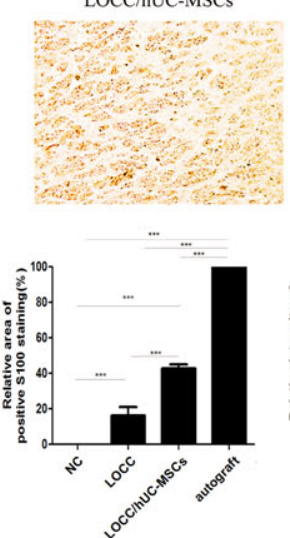

LOCC

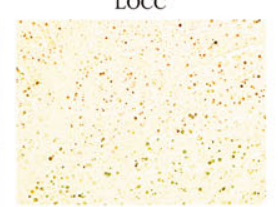

autograf
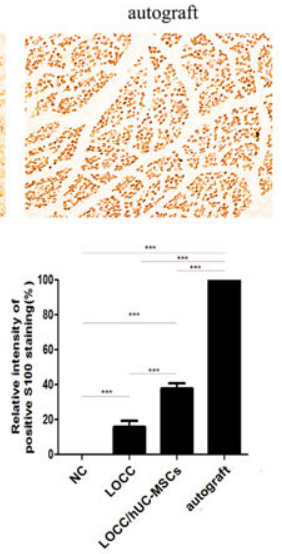

(c)

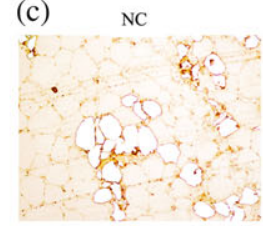

LOCC/hUC-MSCs
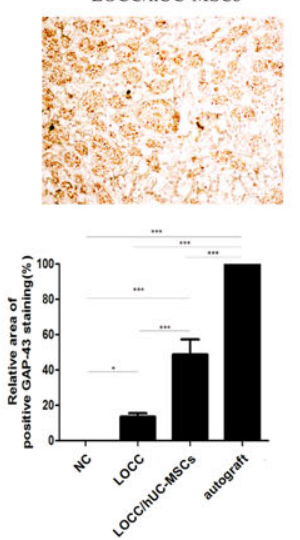

LOCC

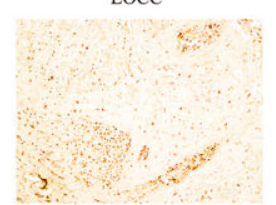

autograft
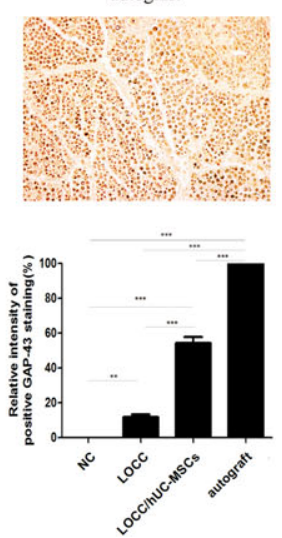

FIGURE 5 Immunohistochemical analysis of the central section of the regenerated nerve 9 months after surgery in the negative control (NC), longitudinally oriented collagen conduit (LOCC), LOCC/human umbilical cord mesenchymal stem cell (hUC-MSC), and autograft groups. Positive immunolabelling for (a) neurofilament (NF), (b) S-100, and (c) growth-associated protein (GAP)-43 in the regenerated nerve tissue. The graph shows the relative areas and intensities of the NF-, S100-, and GAP43-positive staining. The data are presented as the mean \pm standard deviation. ${ }^{*} p<.05$, ${ }^{* *} p<.01$, or ${ }^{* * *} p<.001$, compared with the NC group [Colour figure can be viewed at wileyonlinelibrary.com] 

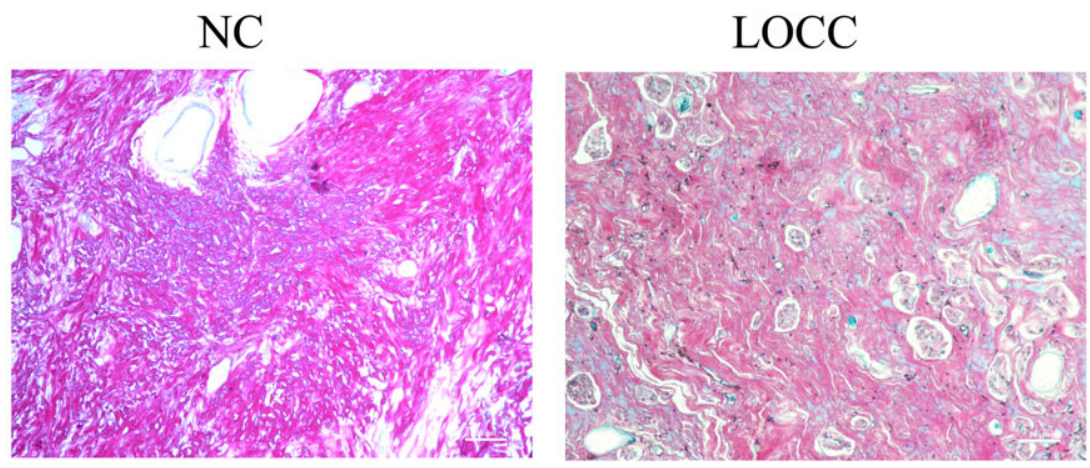

\section{LOCC/hUC-MSCs}
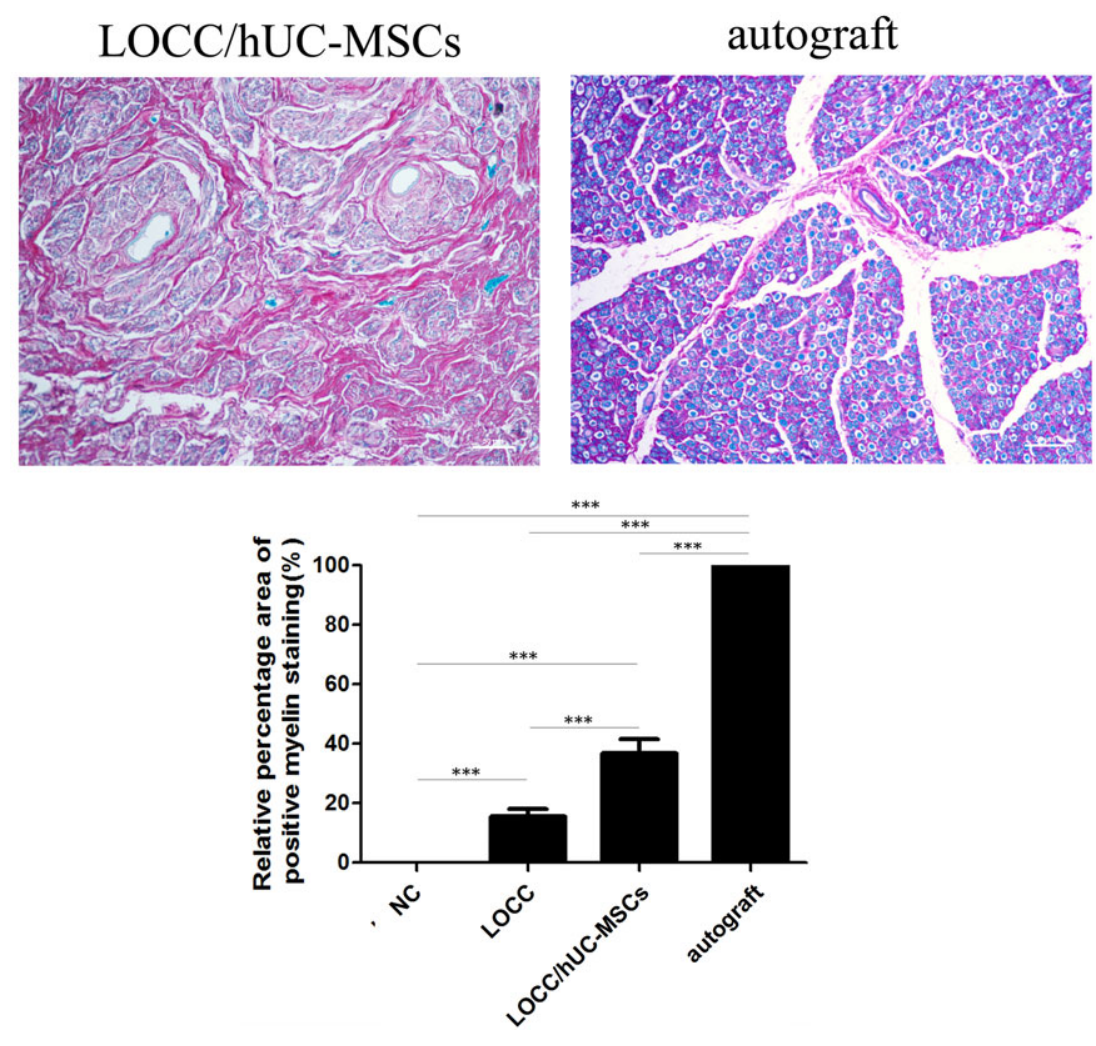

gradually decreased as interstitial fibrosis increased. Figure $8 \mathrm{~b}$ shows the skeletal muscle fibres, which are stained red, and interstitial fibrosis tissue, which is stained white. Gastrocnemius muscle fibres were more abundant in the LOCC/hUC-MSCs group than in the LOCC group (Figure 8b).

\section{4 | DISCUSSION}

Peripheral nerves possess the capacity to regenerate and self-repair after injury, especially when an appropriate micro-environment is provided. Tissue engineering to promote repair following peripheral nerve injury requires the united efforts of biologists, engineers, and physicians towards the common goal of producing functional artificial neural conduits as alternatives to nerve autografts, whether the material is natural or synthetic. For clinical application, tubular nerve guidance channels need to be easy to manufacture, sanitize, and implant into the body.

It has been reported that artificial neural tube conduits can repair peripheral nerve injuries. Various materials have been used to produce artificial neural tubes, such as poly(L-lactic acid), polyglycolic acid-
FIGURE 6 MCOLL histochemical staining of the central portion of the repaired sciatic nerve segment in each group. The graph shows the relative percentages of myelinpositive staining. The data are presented as the mean \pm standard deviation. ${ }^{* * *} p<.001$, compared with the negative control (NC) group. hUC-MSCs = human umbilical cord mesenchymal stem cells; LOCC = longitudinally oriented collagen conduit [Colour figure can be viewed at wileyonlinelibrary.com] collagen, silicone, chitosan, and silk fibrin, although all of these have disadvantages (Lundborg et al., 1997; Matsumoto et al., 2000; Tsai et al., 2009; Wang et al., 2014). To date, there has been a lack of artificial neural tubes that successfully promote nerve regeneration across long lesion gaps for clinical applications. The efficient repair of long peripheral nerve defects caused by trauma or surgery remains a major clinical objective. When the nerve gap exceeds a certain length, a neural tube alone will not improve nerve regeneration, unless support cells or growth factors are added (de Boer et al., 2012; Dong \& Yi, 2010; Kokai, Bourbeau, Weber, McAtee, \& Marra, 2011; Tohill \& Terenghi, 2004). To create an optimal micro-environment for peripheral nerve regeneration, numerous studies have been focused on the incorporation of support cells or growth factors into artificial tissue-engineered neural tubes. In our previous study, we reported that an LOCC combined with neurotrophic factors provides a suitable functional conduit for promoting spinal cord or peripheral nerve regeneration in large animals. When neurotrophic factors are anchored to the LOCC, the resulting nerve conduit effectively promotes neural regeneration(Cui, Lu, et al., 2014; Han et al., 2015). However, the long-term (>6 months) effectiveness of an LOCC containing hUC-MSCs in bridging large 


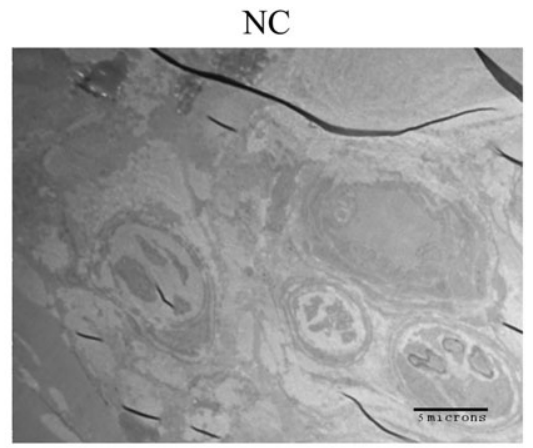

LOCC/hUC-MSCs
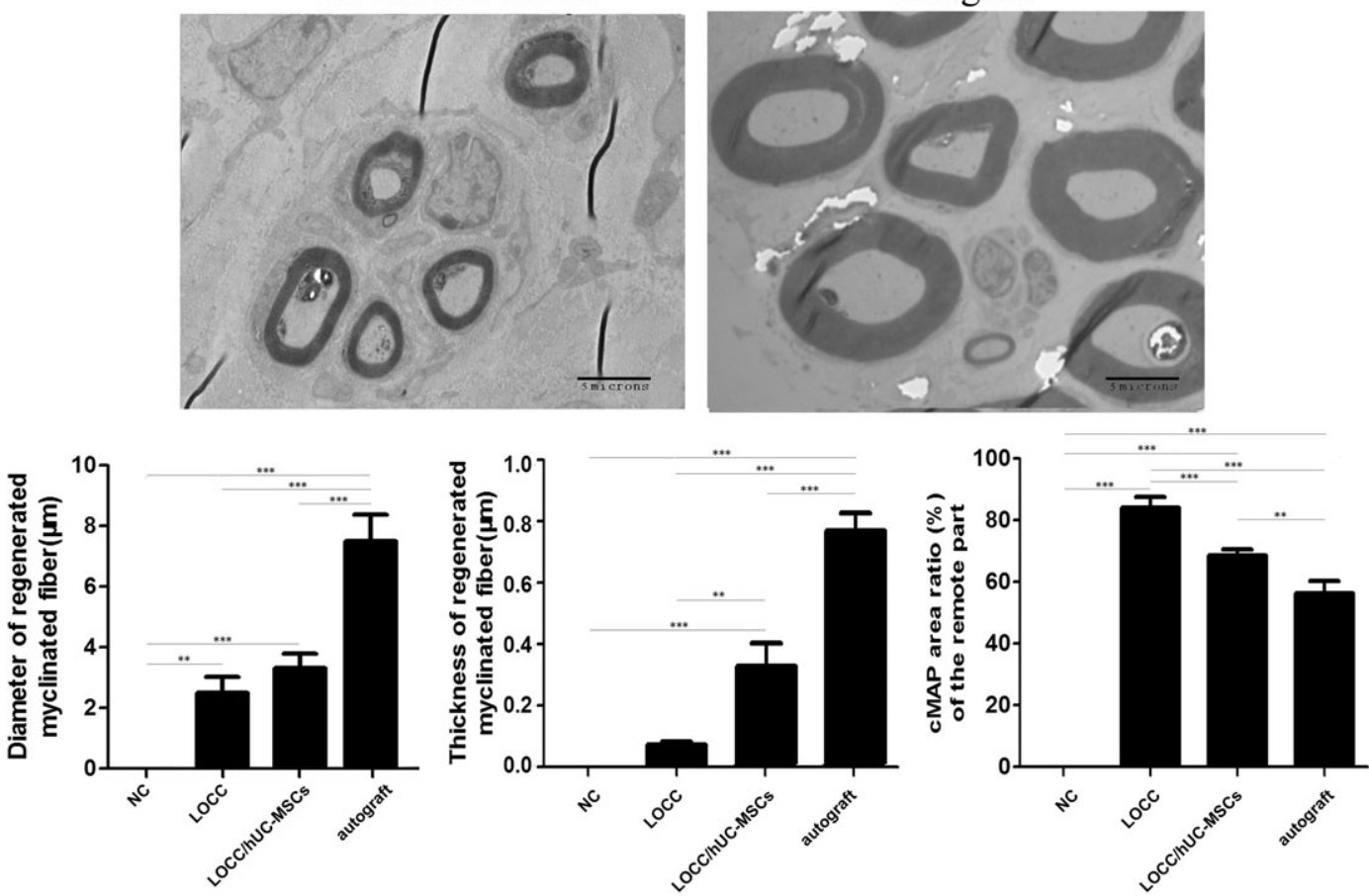

FIGURE 7 Transmission electron microscopic analysis of the myelin sheaths in the middle part of the regenerated nerve. The left graph shows the mean diameter of the nerve fibres. The right graph shows the relative thickness of the regenerated myelin sheaths. The data are presented as the mean \pm standard deviation. ${ }^{* *} p<.01$, or ${ }^{* * *} p<.001$, compared with the negative control (NC) group. hUC-MSCs = human umbilical cord mesenchymal stem cells; LOCC $=$ longitudinally oriented collagen conduit

nerve gaps in large mammals had not yet been assessed. Therefore, in this study, we seeded hUC-MSCs onto an LOCC and assessed their combined effect in a dog model of sciatic nerve transection injury. Compared with rodents, large animal models are more suitable for preclinical evaluation as they better mimic the clinical situation.

To the best of our knowledge, our study is the first to evaluate the effects of an LOCC combined with hUC-MSCs on long-term (9 months) peripheral nerve regeneration in a large animal model. Compared with rodent animals, large animal models are more suitable for the preclinical evaluation study to mimic real clinical situations. We chose the autograft group as the positive control because autografting is the accepted therapeutic strategy for peripheral nerve repair in the clinic. Thus, every artificial nerve conduits should be compared with this standard. The regeneration of peripheral nerves was comprehensively evaluated by histological analysis and electrophysiological assessment. Histological analysis is commonly used to evaluate the outcome of nerve regeneration following repair of peripheral nerve injury. Previous studies showed that GAP-43 is a nervous tissue-specific cytoplasmic protein that is expressed at high levels in neuronal growth cones during axonal regeneration (Carriel et al., 2011; Carriel, Garzon, Campos, et al., 2014). NF is richly distributed in myelinated axons in the nervous system (Perrot, Berges, Bocquet, \& Eyer, 2008), whereas S-100 is mainly localized around axons of peripheral nerves. As a mature Schwann cell marker, S-100 might reflect myelin sheath formation in newborn regenerating nerves (Walsh \& Midha, 2009). As important indexes of axonal regeneration and myelin sheath formation, GAP43, NF, and S100 immunolabelling revealed that the combination of an LOCC and hUC-MSCs effectively stimulates nerve regeneration and nerve fibre myelination over the long term (9 months). The electrophysiological findings suggested that the sciatic nerve defect had been repaired as nerve continuity appeared to be at least partially restored. Because the denervated gastrocnemius muscle undergoes degeneration and fibrosis formation, MT staining is often used to detect morphological changes following nerve injury. We found no obvious pathological changes in the weight or structure of the gastrocnemius muscle fibres on the operated side in the LOCC/hUC-MSC group. 
(a)

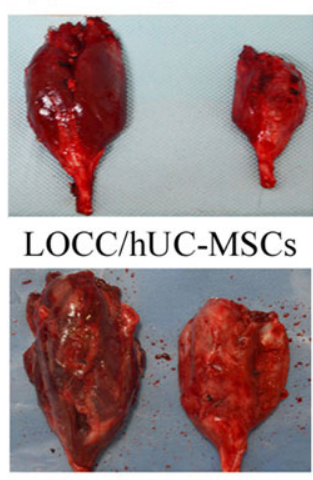

(b)

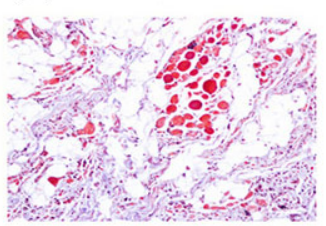

LOCC/hUC-MSCs
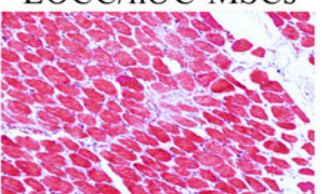

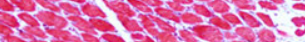

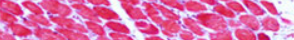

LOCC

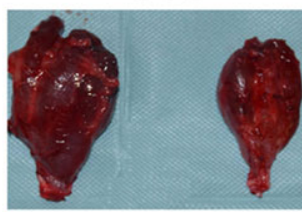

autograft
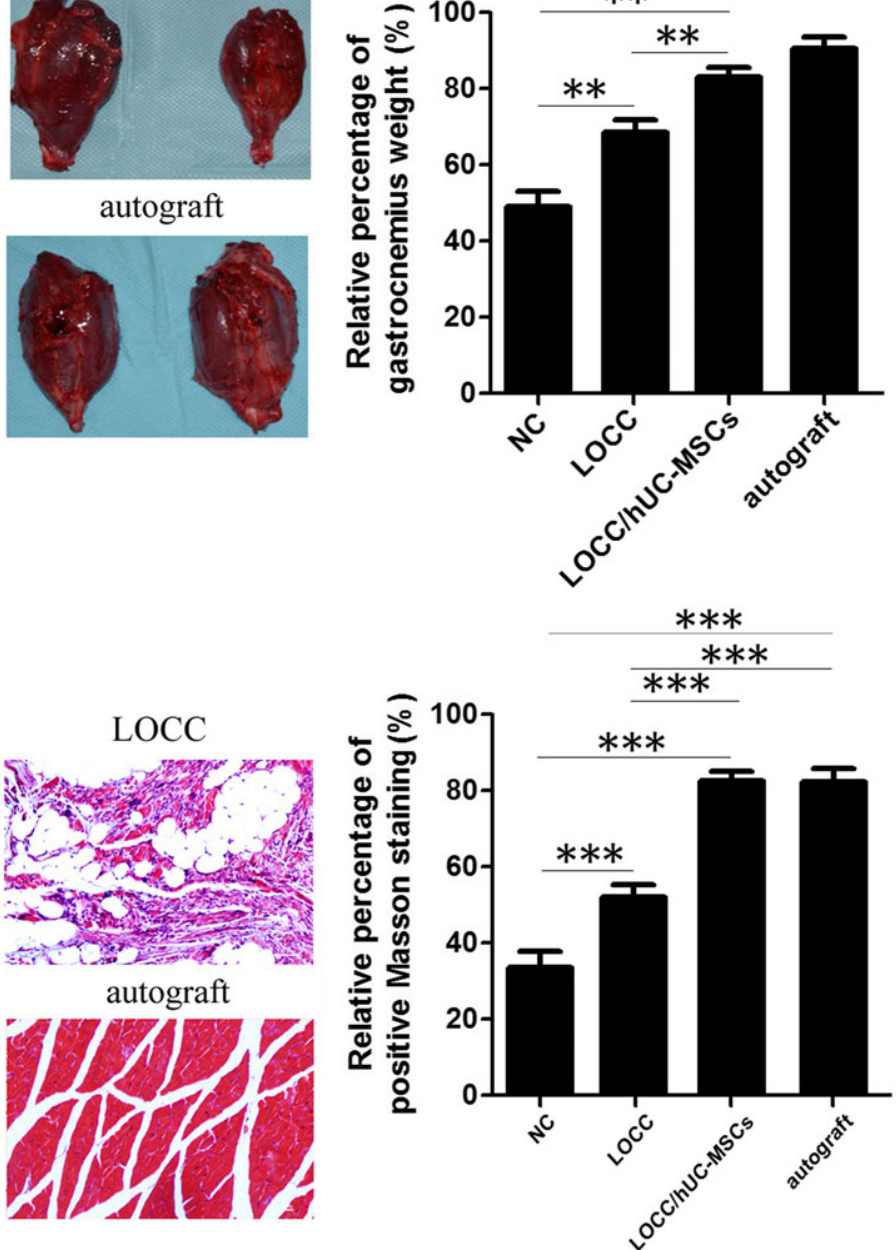

FIGURE 8 (a) Assessment of the gastrocnemius muscles. The gastrocnemius muscles on both sides (injured left and uninjured right) were removed and weighed in all four groups 9 months after surgery. The bar graphs indicate the gastrocnemius muscle weight ratios, which are presented as percentages. (b) Masson trichrome staining showing the morphological changes in the denervated skeletal muscles in each group. The bar graphs indicate the percentages of positive Masson staining. Scale bar: $50 \mu \mathrm{m}$. The data are presented as the mean \pm standard deviation. ${ }^{*} p<.05,{ }^{* *} p<.01$, or ${ }^{* * *} p<.001$, compared with the negative control (NC) group. hUC-MSCs = human umbilical cord mesenchymal stem cells; LOCC = longitudinally oriented collagen conduit [Colour figure can be viewed at wileyonlinelibrary.com]

Numerous studies show that cell-based therapy is a promising strategy for improving peripheral nerve regeneration (Bongso \& Fong, 2013; Caplan \& Dennis, 2006; Carriel et al., 2013; Dong \& Yi, 2010). It is well known that MSCs may serve as support cells within tissueengineered nerve grafts. Moreover, MSCs have achieved considerable success in peripheral nerve repair (Horwitz \& Dominici, 2008; Ringden et al., 2006). In this study, we choose hUC-MSCs as seed cells because they are easily obtained and can be amplified on a large scale in in vitro culture. As an alternative to bone marrow MSCs, hUC-MSCs possess favourable intrinsic features such as self-renewal capacity, hypo-immunogenic and non-tumorigenic properties, and fewer ethical concerns (Bongso \& Fong, 2013; Cui, Li, et al., 2014; Sung et al., 2012). Numerous experimental and clinical trials have demonstrated the efficacy and safety of hUC-MSCs in treating peripheral nerve injury (Dorronsoro \& Robbins, 2013; El Omar et al., 2014; Wang et al., 2013). Recent studies show that undifferentiated hUC-MSCs produce several neurotrophic and growth-related factors, including nerve growth factor, brain-derived neurotrophic factor, p75 neurotrophin receptor, neurotrophin-3, neurotrophin-5, glial cell line-derived neurotrophic factor, and NFs (Caplan \& Dennis, 2006; Dorronsoro \& Robbins, 2013; Fan et al., 2005). The facilitative effect of hUC-MSCs on nerve regeneration can be partly attributed to the secretion of neurotrophic factors. The possible paracrine effects of hUC-MSCs helped to establish a more favourable microenvironment for nerve regeneration.

In this study, we found that the use of an LOCC seeded with hUCMSCs results in greater myelin sheath formation in the middle part of the graft. The functional LOCC may provide good support for the newborn regenerating fibres and guide their growth in the right direction to bridge the nerve gap. As expected, the LOCC/hUC-MSCs combination resulted in better functional recovery compared with the LOCC alone (All the statistical data are listed in the Supporting Information DataS1 and DataS2). Therefore, an LOCC seeded with hUC-MSCs might have therapeutic potential for the treatment of peripheral nerve injuries. However, the enhancement of nerve regeneration in the LOCC/hUCMSCs group was inferior to that in the autologous nerve graft group. 
Therefore, our LOCC requires further improvement before serving as an effective alternative to conventional autografts for the repair of peripheral nerve injuries.

\section{5 | CONCLUSIONS}

The treatment of severe peripheral nerve injuries is a major clinical challenge. The formidable limitations of nerve autografts have spurred the development of tissue-engineered nerve grafts. Our findings here demonstrate that an LOCC containing hUC-MSCs possesses unique biochemical and structural properties that promote nerve regeneration and functional recovery. The method presented here is easily performed without any limitations, complications, or adverse effects. Therefore, it may represent an alternative treatment for peripheral nerve injury.

\section{ACKNOWLEDGEMENTS}

This work was supported by grants from the Key Research Program of the Chinese Academy of Sciences (Grant ZDRW-ZS-2016-2), the National Key Research and Development Project (2016YFC1000800 and 2016YFC1101500), and the Youth Innovation Promotion Association, CAS (2016096). We thank Jie Sun for supplying hUC-MSCs. We thank Bin Wang, Xing Li, and Sufang Han for preparing the nerve conduit. We thank Nuo Wang, Bing Chen, and Yongxiang Fang for their expertise in immunohistochemistry. We thank Piao Wang, Hao Zhao, and Chao Lu for animal surgery and expert animal care. We thank Barry Patel, PhD, from Liwen Bianji, Edanz Group China (www.liwenbianji.cn/ ac), for editing the English text of a draft of this manuscript.

\section{CONFLICT OF INTEREST}

The authors have declared that there is no conflict of interest.

\section{ORCID}

Jianwu Dai (D) http://orcid.org/0000-0002-3379-9053

\section{REFERENCES}

Ao, Q., Fung, C. K., Tsui, A. Y., Cai, S., Zuo, H. C., Chan, Y. S., \& Shum, D. K. (2011). The regeneration of transected sciatic nerves of adult rats using chitosan nerve conduits seeded with bone marrow stromal cell-derived Schwann cells. Biomaterials, 32(3), 787-796.

de Boer, R., Borntraeger, A., Knight, A. M., Hébert-Blouin, M. N., Spinner R. J., Malessy, M. J., ... Windebank, A. J. (2012). Short- and long-term peripheral nerve regeneration using a poly-lactic-co-glycolic-acid scaffold containing nerve growth factor and glial cell line-derived neurotrophic factor releasing microspheres. Journal of Biomedical Materials Research. Part A, 100(8), 2139-2146.

Bongso, A., \& Fong, C. Y. (2013). The therapeutic potential, challenges and future clinical directions of stem cells from the Wharton's jelly of the human umbilical cord. Stem Cell Reviews, 9(2), 226-240.

Cao, J., Xiao, Z., Jin, W., Chen, B., Meng, D., Ding, W., ... Dai, J. (2013). Induction of rat facial nerve regeneration by functional collagen scaffolds. Biomaterials, 34(4), 1302-1310.

Caplan, A. I., \& Dennis, J. E. (2006). Mesenchymal stem cells as trophic mediators. Journal of Cellular Biochemistry, 98(5), 1076-1084.

Carriel, V., Alaminos, M., Garzon, I., Campos, A., \& Cornelissen, M. (2014). Tissue engineering of the peripheral nervous system. Expert Review of Neurotherapeutics, 14(3), 301-318.
Carriel, V., Campos, A., Alaminos, M., Raimondo, S., \& Geuna, S. (2017). Staining methods for normal and regenerative myelin in the nervous system. Methods in Molecular Biology, 1560, 207-218.

Carriel, V., Garrido-Gomez, J., Hernandez-Cortes, P., Garzón, I., GarcíaGarcía, S., Sáez-Moreno, J. A., ... Alaminos, M. (2013). Combination of fibrin-agarose hydrogels and adipose-derived mesenchymal stem cells for peripheral nerve regeneration. Journal of Neural Engineering, 10(2), 026022.

Carriel, V., Garzon, I., Alaminos, M., \& Campos, A. (2011). Evaluation of myelin sheath and collagen reorganization pattern in a model of peripheral nerve regeneration using an integrated histochemical approach, Histochemistry and Cell Biology, 136(6), 709-717.

Carriel, V., Garzon, I., Alaminos, M., \& Cornelissen, M. (2014). Histological assessment in peripheral nerve tissue engineering. Neural Regeneration Research, 9(18), 1657-1660.

Carriel, V., Garzon, I., Campos, A., Comelissen, M., \& Alaminos, M. (2014). Differential expression of GAP-43 and neurofilament during peripheral nerve regeneration through bio-artificial conduits. Journal of Tissue Engineering and Regenerative Medicine.

Cui, B., Li, E., Yang, B., \& Wang, B. (2014). Human umbilical cord blood-derived mesenchymal stem cell transplantation for the treatment of spinal cord injury. Experimental and Therapeutic Medicine, 7(5), 1233-1236.

Cui, Y., Lu, C., Meng, D., Xiao, Z., Hou, X., Ding, W., ... Dai, J. (2014). Collagen scaffolds modified with CNTF and bFGF promote facial nerve regeneration in minipigs. Biomaterials, 35(27), 7819-7827.

Dong, M. M., \& Yi, T. H. (2010). Stem cell and peripheral nerve injury and repair. Facial Plastic Surgery, 26(5), 421-427.

Dorronsoro, A., \& Robbins, P. D. (2013). Regenerating the injured kidney with human umbilical cord mesenchymal stem cell-derived exosomes. Stem Cell Research \& Therapy, 4(2), 39.

Dwyer, T., Willett, T. L., Dold, A. P., Petrera, M., Wasserstein, D., Whelan, D. B., \& Theodoropoulos, J. S. (2016). Maximum load to failure and tensile displacement of an all-suture glenoid anchor compared with a screw-in glenoid anchor. Knee Surgery, Sports Traumatology, Arthroscopy, 24(2), 357-364.

El Omar, R., Beroud, J., Stoltz, J. F., Menu, P., Velot, E., \& Decot, V. (2014). Umbilical cord mesenchymal stem cells: The new gold standard for mesenchymal stem cell-based therapies? Tissue Engineering. Part $B$, Reviews, 20(5), 523-544.

Fan, C. G., Zhang, Q. J., Tang, F. W., Han, Z. B., Wang, G. S., \& Han, Z. C. (2005). Human umbilical cord blood cells express neurotrophic factors. Neuroscience Letters, 380(3), 322-325.

Faroni, A., Mobasseri, S. A., Kingham, P. J., \& Reid, A. J. (2015). Peripheral nerve regeneration: Experimental strategies and future perspectives. Advanced Drug Delivery Reviews, 82-83, 160-167.

Grasman, J. M., Pumphrey, L. M., Dunphy, M., Perez-Rogers, J., \& Pins, G. D. (2014). Static axial stretching enhances the mechanical properties and cellular responses of fibrin microthreads. Acta Biomaterialia, 10(10), 4367-4376.

Gu, X., Ding, F., \& Williams, D. F. (2014). Neural tissue engineering options for peripheral nerve regeneration. Biomaterials, 35(24), 6143-6156.

Guo, Z. Y., Sun, X., Xu, X. L., Zhao, Q., Peng, J., \& Wang, Y. (2015). Human umbilical cord mesenchymal stem cells promote peripheral nerve repair via paracrine mechanisms. Neural Regeneration Research, 10(4), 651-658.

Han, S., Wang, B., Jin, W., Xiao, Z., Li, X., Ding, W., ... Dai, J. (2015). The linear-ordered collagen scaffold-BDNF complex significantly promotes functional recovery after completely transected spinal cord injury in canine. Biomaterials, 41, 89-96.

Horwitz, E. M., \& Dominici, M. (2008). How do mesenchymal stromal cells exert their therapeutic benefit? Cytotherapy, 10(8), 771-774.

Isaacs, J., Mallu, S., \& Batchelor, M. (2014). Modification of commercially available image analysis software for semi-automated qualitative 
analysis of axon regeneration and myelination in the rat sciatic nerve. Journal of Neuroscience Methods, 233, 45-49.

Kao, S. Y., Shyu, J. F., Wang, H. S., Lin, C. H., Su, C. H., Chen, T. H., ... Tsai, P. J. (2015). Comparisons of differentiation potential in human mesenchymal stem cells from Wharton's jelly, bone marrow, and pancreatic tissues, Stem Cells International, 2015, 306158, 1, 10.

Kokai, L. E., Bourbeau, D., Weber, D., McAtee, J., \& Marra, K. G. (2011). Sustained growth factor delivery promotes axonal regeneration in long gap peripheral nerve repair. Tissue Engineering. Part A, 17(9-10), 1263-1275.

Lee, J. C., Kim, K. C., Yang, Y. S., Oh, W., Choi, S. J., Choe, S. Y., \& Hong, Y. M. (2014). Microarray analysis after umbilical cord blood derived mesenchymal stem cells injection in monocrotaline-induced pulmonary artery hypertension rats. Anat Cell Biol, 47(4), 217-226.

Li, R., Wu, J., Lin, Z., Nangle, M. R., Li, Y., Cai, P., ... Xiao, J. (2017). Single injection of a novel nerve growth factor coacervate improves structural and functional regeneration after sciatic nerve injury in adult rats. Experimental Neurology, 288, 1-10.

Lu, C., Meng, D., Cao, J., Xiao, Z., Cui, Y., Fan, J., ... Dai, J. (2015). Collagen scaffolds combined with collagen-binding ciliary neurotrophic factor facilitate facial nerve repair in mini-pigs. Journal of Biomedical Materials Research. Part A, 103(5), 1669-1676.

Lundborg, G., Dahlin, L., Dohi, D., Kanje, M., \& Terada, N. (1997). A new type of "bioartificial" nerve graft for bridging extended defects in nerves. Journal of Hand Surgery (British), 22(3), 299-303.

Ma, F., Xiao, Z., Chen, B., Hou, X., Dai, J., \& Xu, R. (2014). Linear ordered collagen scaffolds loaded with collagen-binding basic fibroblast growth factor facilitate recovery of sciatic nerve injury in rats. Tissue Engineering. Part A, 20(7-8), 1253-1262.

Matsumoto, K., Ohnishi, K., Kiyotani, T., Sekine, T., Ueda, H., Nakamura, T., ... Shimizu, Y. (2000). Peripheral nerve regeneration across an 80-mm gap bridged by a polyglycolic acid (PGA)-collagen tube filled with laminin-coated collagen fibers: A histological and electrophysiological evaluation of regenerated nerves. Brain Research, 868(2), 315-328.

Perrot, R., Berges, R., Bocquet, A., \& Eyer, J. (2008). Review of the multiple aspects of neurofilament functions, and their possible contribution to neurodegeneration. Molecular Neurobiology, 38(1), 27-65.

Ringden, O., Uzunel, M., Rasmusson, I., Remberger, M., Sundberg, B., Lönnies, H., ... Le Blanc, K. (2006). Mesenchymal stem cells for treatment of therapy-resistant graft-versus-host disease. Transplantation, 81(10), 1390-1397.

Sun, J., Zhao, Y., Li, Q., Chen, B., Hou, X., Xiao, Z., \& Dai, J. (2016). Controlled release of collagen-binding SDF-1alpha improves cardiac function after myocardial infarction by recruiting endogenous stem cells. Scientific Reports, 6, 26683.

Sung, M. A., Jung, H. J., Lee, J. W., Lee, J. Y., Pang, K. M., Yoo, S. B., ... Lee, J. H. (2012). Human umbilical cord blood-derived mesenchymal stem cells promote regeneration of crush-injured rat sciatic nerves. Neural Regeneration Research, 7(26), 2018-2027.

Tohill, M., \& Terenghi, G. (2004). Stem-cell plasticity and therapy for injuries of the peripheral nervous system. Biotechnology and Applied Biochemistry, 40(Pt 1), 17-24.

Tsai, P. C., Fu, T. W., Chen, Y. M., Ko, T. L., Chen, T. H., Shih, Y. H., ... Fu, Y. S. (2009). The therapeutic potential of human umbilical mesenchymal stem cells from Wharton's jelly in the treatment of rat liver fibrosis. Liver Transplantation, 15(5), 484-495.

Wakao, S., Matsuse, D., \& Dezawa, M. (2014). Mesenchymal stem cells as a source of Schwann cells: Their anticipated use in peripheral nerve regeneration. Cells, Tissues, Organs, 200(1), 31-41.

Walsh, S., \& Midha, R. (2009). Use of stem cells to augment nerve injury repair. Neurosurgery, 65(4 Suppl), A80-A86.

Wang, L., Cong, X., Liu, G., Zhou, J., Bai, B., Li, Y., ... Liu, Y. (2013). Human umbilical cord mesenchymal stem cell therapy for patients with active rheumatoid arthritis: Safety and efficacy. Stem Cells and Development, 22(24), 3192-3202.

Wang, H., Qiu, X., Ni, P., Qiu, X., Lin, X., Wu, W., ... Ma, L. (2014). Immunological characteristics of human umbilical cord mesenchymal stem cells and the therapeutic effects of their transplantion on hyperglycemia in diabetic rats. International Journal of Molecular Medicine, 33(2), 263-270.

Zarbakhsh, S., Goudarzi, N., Shirmohammadi, M., \& Safari, M. (2016). Histological study of bone marrow and umbilical cord stromal cell transplantation in regenerating rat peripheral nerve. Cell Journal, 17(4), 668-677.

\section{SUPPORTING INFORMATION}

Additional Supporting Information may be found online in the supporting information tab for this article.

Data S1. The statistical analysis data of means \pm SD for all the qualitative statements in all 4 groups.

Data S2. The statistical analysis data of $95 \%$ confidence interval and $p$ value for all the pairwise comparison.

How to cite this article: Cui Y, Yao Y, Zhao Y, et al. Functional collagen conduits combined with human mesenchymal stem cells promote regeneration after sciatic nerve transection in dogs. J Tissue Eng Regen Med. 2018;12:1285-1296. https:// doi.org/10.1002/term.2660 